\title{
PERFORMANCE COMPARISON OF THE DIFFERENT STREAMS IN A TCP BOTTLENECK LINK IN THE PRESENCE OF BACKGROUND TRAFFIC IN A DATA CENTER
}

\author{
Vilma Tomço, ${ }^{1}$ Aleksandër Xhuvani
}

\begin{abstract}
The purpose of this work is the evaluation of the performance of TCP streams when the link that causes a bottleneck is also shared by the background traffic. Standard TCP is the most used protocol which sustains the majority of Internet traffic. Nevertheless, TCP manifests some problems when using almost all of the available bandwidth. Based on these problems, there are recognized different versions of TCP. The aim of this paper is to test the conflict between TCP flows bandwidth allocation. We have implemented a network which includes FTP traffic and background traffic. The TCP flows are simulated so that they begin transmitting at different times. Background traffic is added besides the TCP flows so that we approach a real network model. Besides studying how the TCP flows compete with each other, we will evaluate if the background traffic has an impact on the behavior of TCP flows and if it influences how the available bandwidth is shared equally among flows. We compare standard TCP Tahoe and TCP Reno, which do not differ much between each-other, but use different algorithms so through simulation we will evaluate the changes in the bandwidth they use, even though we expect the TCP flows to be more aggressive in getting bandwidth from other TCP flows.
\end{abstract}

JEL Classification number: O33; DOI: http://dx.doi.org/10.12955/cbup.v5.1102

UDC Classification: 654

Keywords: components; TCP stream, FTP Traffic, TCP Tahoe, TCP Reno;

\section{Introduction}

TCP and UDP are the two protocols of the transport layer used in Internet network. TCP is a reliable connection-oriented protocol, which guarantees the sending of the packets from source to destination in the order they were sent (Chohan, 2006). UDP is an unreliable, connectionless protocol, so the best effort protocol which uses no mechanism to handle packet loss or managing out of order received packets. TCP is a congestion control protocol which controls the rate at which packets are transmitted between sender and receiver (it slows down packet sending speed when it detects that the network is overloaded). The purpose of the UDP protocol is to send data as fast as possible. Many implementations of TCP are realized, and for each of these implementations, different studies over their impact on network utilization are done. Based on this two protocols we will study the behavior of TCP flows which show an aggressive approach in getting the bandwidth, and UDP which transmits at a constant rate regardless of other traffic on the network. We have used the NS2 simulator (Issariyakul, 2009) to evaluate the differences between the TCP flows, and also to study the impact of the background traffic. In this paper, we will look through providing a Network Random Input, the analysing of Initial Conditions, the Mathematical Model (used algorithms), the realization of some Simulations (Yuvaraju et al., 2010), the Graphic Representation of Flow Comparison, the Calculation of the Confidence Interval and at the Conclusions from the finished simulations (Cho, 2006). At the end of the paper, there are included the references that we have referred to during our work.

\section{Network random input}

The network model we have used is composed of 10 nodes, based on the Dumbo-Bell typology that consists of only one bottleneck link which is shared between some streams. In the network, we have included two TCP flows, one UDP flow with CBR traffic and another UDP flow with VBR traffic where both UDP streams are in the background.

To test the network behavior, so that it approaches the real network where the traffic changes over time, we have included the different elements. First, we have involved background traffic; second, we have included VBR traffic which changes over time. We also have used the exponential method of generating the traffic, through which we do not generate traffic all the time, but there are periods of time when we generate traffic (burst time) and periods of time when we do not generate traffic (idle time). Third, to get different results each time we simulate the network we use a random number generator method which provides us random input for each simulation we do.

\footnotetext{
${ }^{1}$ University of Tirana Computer Science \& Applied Mathematics, Tiranë, Albania, vimatster@gmail.com

${ }^{2}$ Polytechnic University, Tiranë, Albania, axhuvani@yahoo.com
} 


\section{Initial condition}

Based on our network typology we distinguish four streams which share the bottleneck link between the nodes 4 and 5 . The bottleneck link is $10 \mathrm{Mbps}$ full-duplex and with a queue size of 10 packets while the other links have a queue size of 100 packets.

- We create traffic between nodes 0 and 6 (TCP Reno). For the TCP link, we use the maximum size of 100 packets for the congestion window and we take a 512 byte packet size, as we have an Ethernet link and that's the packet size which can be transmitted in the network based on RFC 895 and it is also the standard packet size used by UDP. We use the minimum timeout of 0.2 seconds, and we set the first UDP flow's starting time at 5.0 seconds and the ending time at 90.0 seconds.

- We create FTP traffic between nodes 3 and 9 (TCP Tahoe). We use the same parameters for this flow as the one above. In this way, we evaluate the flows under the same conditions. This flow begins to transmit later than the first one, starting at 10.0 seconds and ending at 90.0 seconds.

- We create CBR traffic between nodes 1 and 7. The CBR traffic generates packets at the speed of 100 packets/sec. The CBR traffic source starts at 15.0 seconds and ends at 60.0 seconds. CBR traffic does not transmit actual data but instead informs the UDP agent that has data to transmit, and the agent creates packets and sends the data. We use a simplex link as UDP does not have a congestion control mechanism.

- We create 1 Mbps VBR traffic between nodes 2 and 8 . We use the exponential on/off method to generate traffic, and we set the "On" period to 150ms and the "Off" period to $100 \mathrm{~ms}$. The link which is used is a simplex one. The packet size is same as the one used in TCP and CBR. VBR traffic starts at 20.0 seconds and ends at the 70.0 seconds mark.

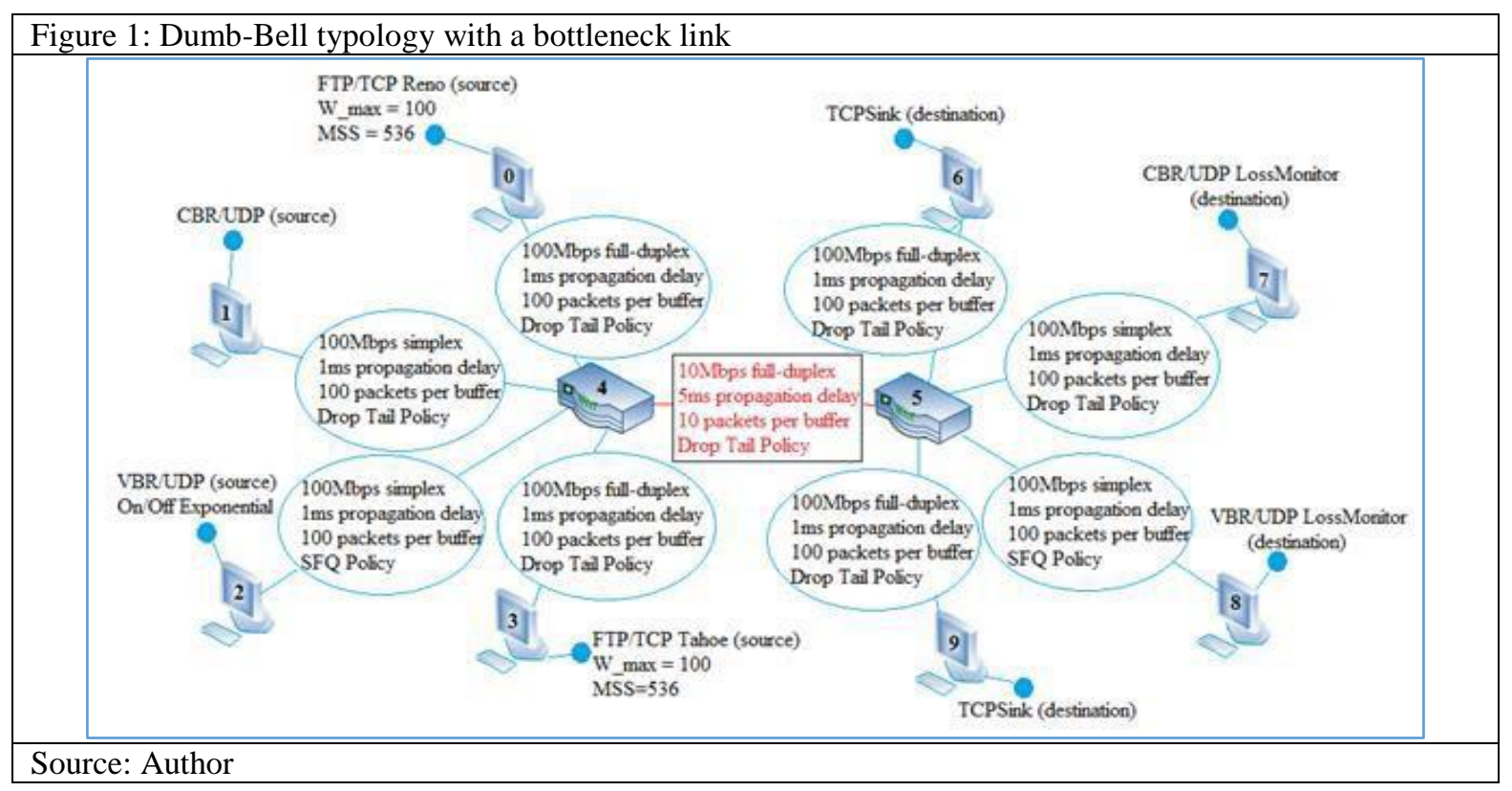

Network typology with 10 nodes:

$>$ The nodes 4 and 5 create the bottleneck link

$>$ The nodes 0 and 3 generate FTP traffic (source nodes)

$>$ The nodes 1 and 2 generate CBR and VBR traffic (source nodes)

$>$ The nodes $6,7,8,9$ are destination nodes

We set the bandwidth to 10 Mbps full- duplex for the bottleneck link to simulate a 10 BaseT Ethernet link. The queue size is 10 packets smaller than the queue size of other links to create in this way the bottleneck and the buffer should have an appropriate size as the congestion avoidance mechanism brings throughput oscillation. Over buffered routers increase latency when we have congestion, whereas under buffered routers bring more problems because packet forwarding generates throughput oscillation. 
For all the other network links we set the capacity to 100 Mbps full-duplex to simulate a 100BaseTX Ethernet link, and we set the queue size to 100 packets. For most of the links, we use the DropTail algorithm for the queue (FIFO) whereas for the n2-n4 and n5-n8 links we use the SFQ algorithm to provide various network conditions.

In the simulation we will perform, a Dumb- Bell typology with 2 TCP sessions is used which shares only a bottleneck link between two routers. Dumb-Bell typology with a congested link allows us to study the network traffic and it simulates a bottleneck link where the TCP sessions are transmitted on the internet. Along with the TCP streams, we have the UDP streams which are placed in the background and cause congestion and TCP packet loss.

\section{Mathematical model}

The UDP protocol does not implement any congestion avoidance mechanism contrary to the TCP protocol which has congestion control mechanism (Cho, 2006). Standard TCP Tahoe is one of the earliest implementations which use the go-back-n model to control network congestion.

TCP Tahoe is based on the algorithms:

1) SlowStart

2) CongestionAvoidance

3) FastRetransmit

TCP Reno is similar to TCP Tahoe, but they differ because TCP Reno has an additional algorithm which is FastRecovery (Ha et al., 2011). During the slow start phase, the congestion window increases with one for each acknowledge (ACK) message received, so the congestion window grows exponentially. The slow start phase continues until the congestion window is equal to or greater than the threshold. Once this condition is true, the next phase begins which is congestion avoidance. During the congestion avoidance phase, instead of increasing the window size with one each time we receive a ACK message, and the congestion avoidance algorithm increases the window size with one for each RTT (Round-Trip delay Time), so in this way, the congestion window size has a linear growth.

In the fast retransmit phase, when we receive a certain number of duplicated ACK for the same packet, the sender retransmits the packet without waiting for the timer to expire.

Fast recovery works during those cases when a certain number of duplicated ACK (threshold usually is set to 3 ) is received. Just as during fast retransmit, the sender retransmits the lost packets but instead of a slow start the congestion window is decreased by half, and then it counts the duplicated ACK to determine when it should send packets again. The window used by the sender:

min (awin, cwin + ndup)

- awin: receiver window

- cwin; sender congestion window

- ndup: the number of duplicated ACK (stays at 0 until the number of duplicated ACK reaches threshold)

Referring RFC 2581 related to TCP Congestion Control we could explain the mathematical model based on the mentioned algorithms. Specifications:

- SEGMENT: each TC/IP data or ACK packet

- SENDER MAXIMUM SEGMENT SIZE (SMSS): the maximum size of the segment that the sender can transmit

- RECIEVER MAXIMUM SEGMENT SIZE (RMSS): the maximum size of the segment that the receiver can accept

- FULL-SIZED SEGMENT: the segment which has the maximum number of data bytes that is allowed

- RECEIVER WINDOW (rwnd): the window that the receiver uses most

- CONGESTION WINDOW (cwnd):

The state variables that limit the amount of data that a TCP sender can transmit. At a certain time, a TCP sender may not send data with a greater sequential number than the sequential number confirmed and the minimum of cwnd and rwnd

- INITIAL WINDOW (IW): the size of cwnd after three-way-handshake has finished 
- LOSS WINDOW (LW): the size of cwnd after the TCP sender detects loss of packets using a retransmission timer

- RESTART WINDOW (RW): the size of cwnd after the TCP sender again begins the transmission after an idle time (if the slow start algorithm is used)

- FLIGHT SIZE: number of data which are sent but not acknowledged yet.

The cwnd variable is a limit at the sender side, in the number of data the sender can transmit before getting the ACK. The rwnd variable is a limit at the receiver side, in the delayed number of data. Minimum size of cwnd and rwnd manage the transmission of the data; the Slow-start algorithm is used before the transmission or after the recovery of lost packets from retransmission timer, to inspect the network in determining the available bandwidth. IW, cwnd initial size, it should be smaller than or equal to $2 *$ SMSS byte and it should not be greater than 2 segments.

The initial value of the ssthresh (slow-start threshold) can be a random value and it can be reduced as a response to congestion. The slow-start algorithm is used when cwnd ssthresh, and the congestion avoidance algorithm is used when cwnd>ssthresh. The sender can use each of them when cwnd is equal to ssthresh. During slow-start, TCP increments cwnd with at least a SMSS byte for each received ACK that confirms the data send. During congestion avoidance, cwnd is incremented with 1 for each RTT. Slow-start ends when cwnd exceeds ssthresh or when congestion is detected. Congestion avoidance ends when congestion is detected.

$$
\text { cwnd }+=\text { SMSS*SMSS/cwnd (1) }
$$

When a TCP sender detects segment loss using the retransmission timer, the value of ssthresh should be set to:

ssthresh $=\max ($ FlightSize/2, $2 *$ SMSS) (2)

The TCP sender should use the fast retransmit algorithm to detect and repair the loss, based on the ACK duplicated packets. After receiving 3 duplicated ACK, the TCP performs a retransmission for the missing segment, without waiting for the retransmission timer to expire. Fast retransmit and fast recovery algorithms can be implemented together:

1. When the third duplicated ACK is received, ssthresh is set to the value given by equation (2)

2. The lost segment is retransmitted and cwnd is set to ssthresh $+3^{*}$ SMSS. This affects cwnd in three segments which are away from the network and are in the receiver buffer.

3. For each duplicated ACK received, cwnd increments from SMSS. This artificially increments cwnd.

4. A segment is transmitted if it is allowed from the new value of cwnd used by the receiver.

5. When another ACK is received to confirm the new data, cwnd is set to ssthresh. This is called window deflation.

\section{Experimental phase}

To reach conclusions about how the TCP streams compete with each other in the utilization of the bandwidth that is being also shared from the traffic background we realize a total of 10 simulations. Given that the results obtained from each simulation differ, then for all the performed simulations, we realize superposition of graphs to see if they fall almost in the same footprint and to confirm with certainty the received outcome. By all performed simulations we will evaluate how much and what proportion of the available bandwidth occupies each stream and then we will give an overall average of bandwidth utilization from each stream. Below we provide graphical presentations obtained by NS2 to show the different results of realized simulations.

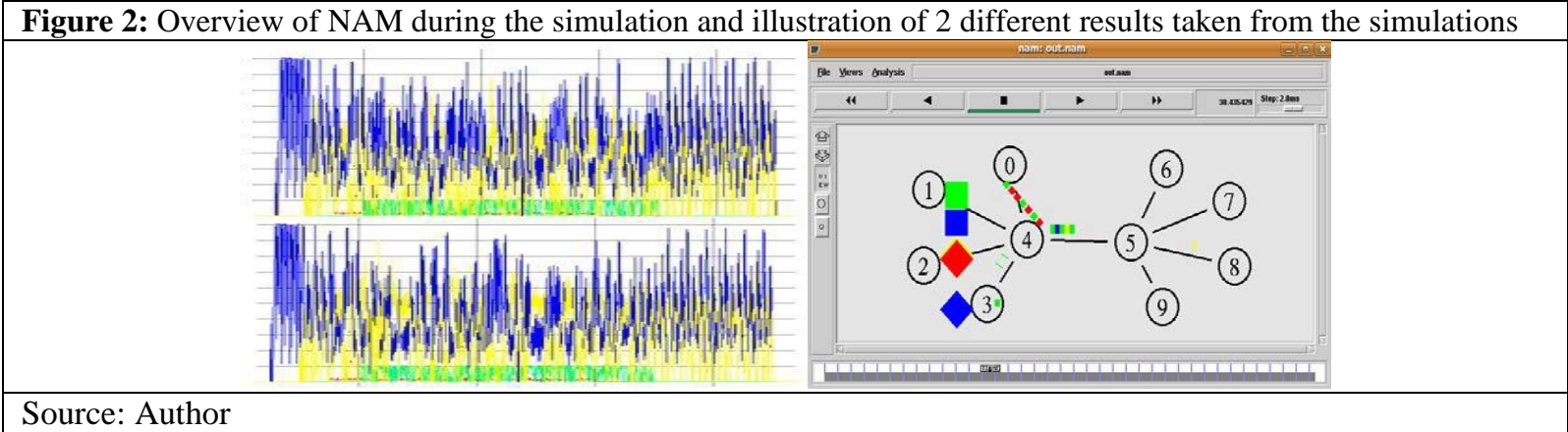




\section{Representation of streams comparison}

The purpose of this work is measuring and analysing the performance of TCP Reno and TCP Tahoe (Mo et al., 1998). The study is done in the presence of background traffic. We will conduct a comparison of TCP streams by evaluating the bandwidth that they allocate (also considering other streams). Regarding the graphics taken from simulations we see that streams which compete aggressively among themselves to exploit as much bandwidth are TCP streams. TCP Reno stream provides more bandwidth than standard TCP Tahoe stream. Meanwhile in cases when in the network appear all types of streams, such as TCP streams and those in the background like VBR or CBR, the bandwidth used by each TCP stream decreases. To give a clearer idea of graphical presentations we initially represent streams separately and then give a graphical representation of all streams that are on the network and compete for bandwidth, evaluating the bandwidth size that each stream occupies.

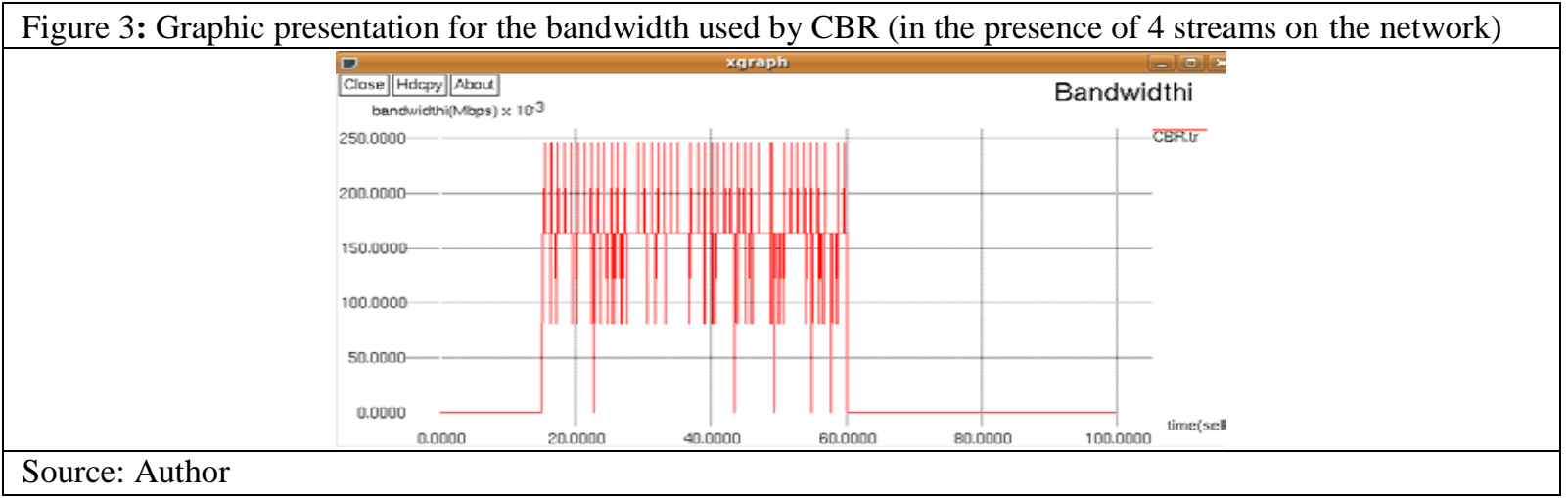

So, as we see from the graph for CBR, the bandwidth that CBR use is very low compared with the size of the link. The maximum value that CBR can achieve in the utilization of the link's bandwidth is $250 \mathrm{kbps}$, and the minimum value is $90 \mathrm{kbps}$. The nominal bandwidth is $170 \mathrm{kbps}$, and this relates to the fact that CBR transmits packages with constant speed and does not show any aggressiveness in obtaining bandwidth. On the other hand, this is related to the fact that UDP does not use any mechanism for congestion.

\begin{tabular}{|l|l|l|}
\hline Figure 4: Graphic presentation for the bandwidth used by VBR (in the presence of 4 streams on network) \\
\hline & \multicolumn{1}{|c|}{ Bandwidthi } \\
\hline
\end{tabular}

Compared with the case of CBR, VBR represents more fluctuations in bandwidth, and this is related to the fact that VBR transmits the packages with different speed. The size of the bandwidth that VBR allocates reaches the maximum value at $1 \mathrm{Mbps}$. Unlike CBR where we used the mechanism Drop Tail, in the case of VBR we use the GFS mechanism.

From the graph obtained for the bandwidth used by TCP Reno, we see that at the very beginning of the simulation when on the network none of the traffic sources had net started any transmission, TCP Reno uses all the potential capacity of the 10Mbps bottleneck link. With the emergence of other traffic on the network, the bandwidth used by TCP Reno decreases, as TCP Reno should share the link- although at certain moments when it can find space in the link, it tends to use it aggressively. It is noted that the average of the values connected with the bandwidth used by TCP Reno is roughly 45 Mbps. 

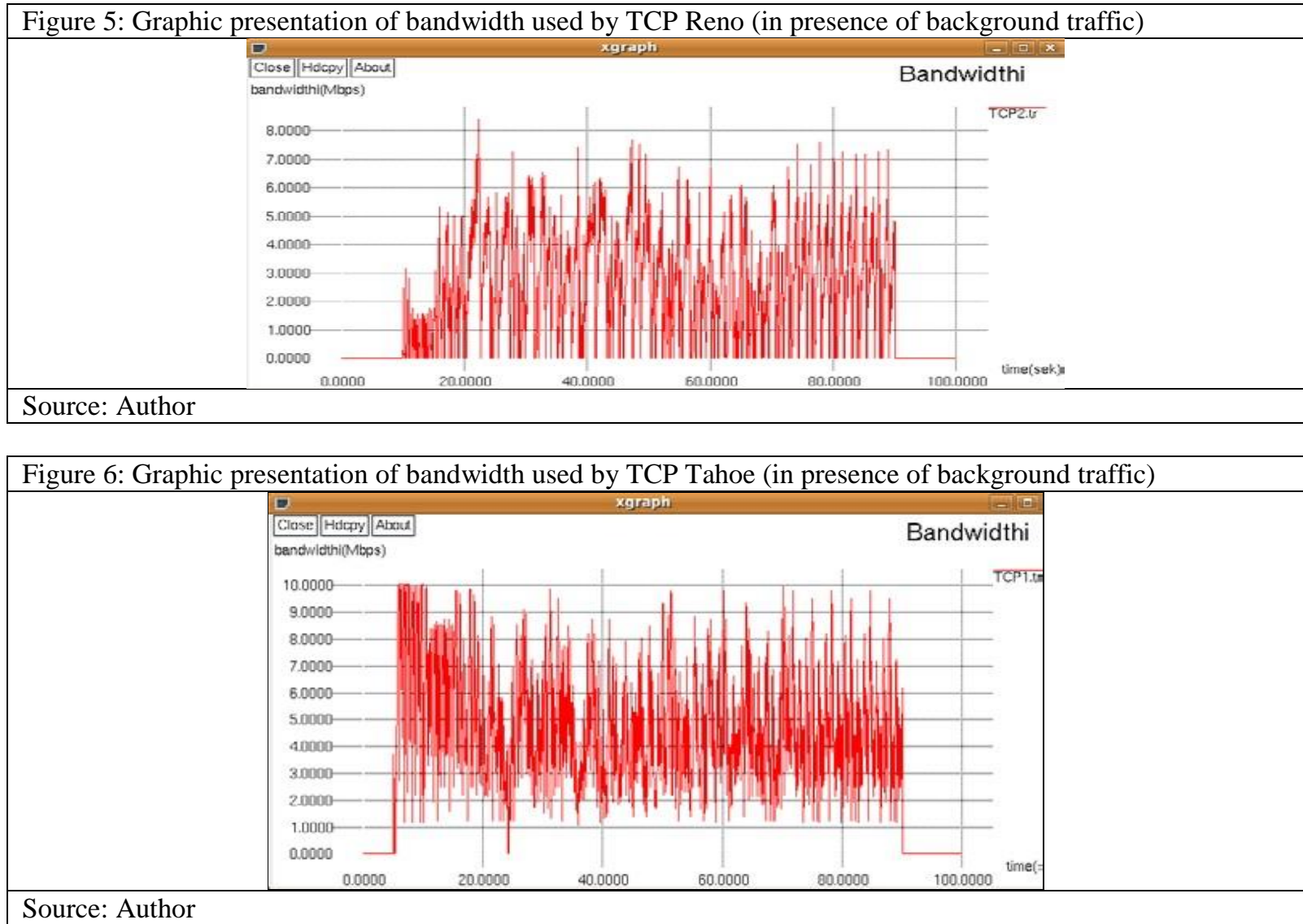

Just as we affirm theoretically that TCP Reno was an improvement on TCP Tahoe and it should be expected to have a low utilization of bandwidth, from the graph given above we confirm such a thing. TCP Tahoe does not show the same aggressiveness as TCP Reno therefore never fails to utilize the full capacity of the maximum bottleneck link. The maximum value of bandwidth that TCP Tahoe can achieve to utilize is $8 \mathrm{Mbps}$. Meanwhile the approximate average value of the average bandwidth that is being used is 2-3 Mbps, which is a result lower than the bandwidth used by TCP Reno.

In the following charts, we will provide graphical presentations of the TCP streams in the presence of background traffics. What is normally expected as a result is that in the presence of background traffic the used bandwidths by each TCP stream are expected to decrease, because the bottleneck link is shared by 4 streams. Normally TCP will remain aggressive in the link not only against the background traffic, but also against each other.

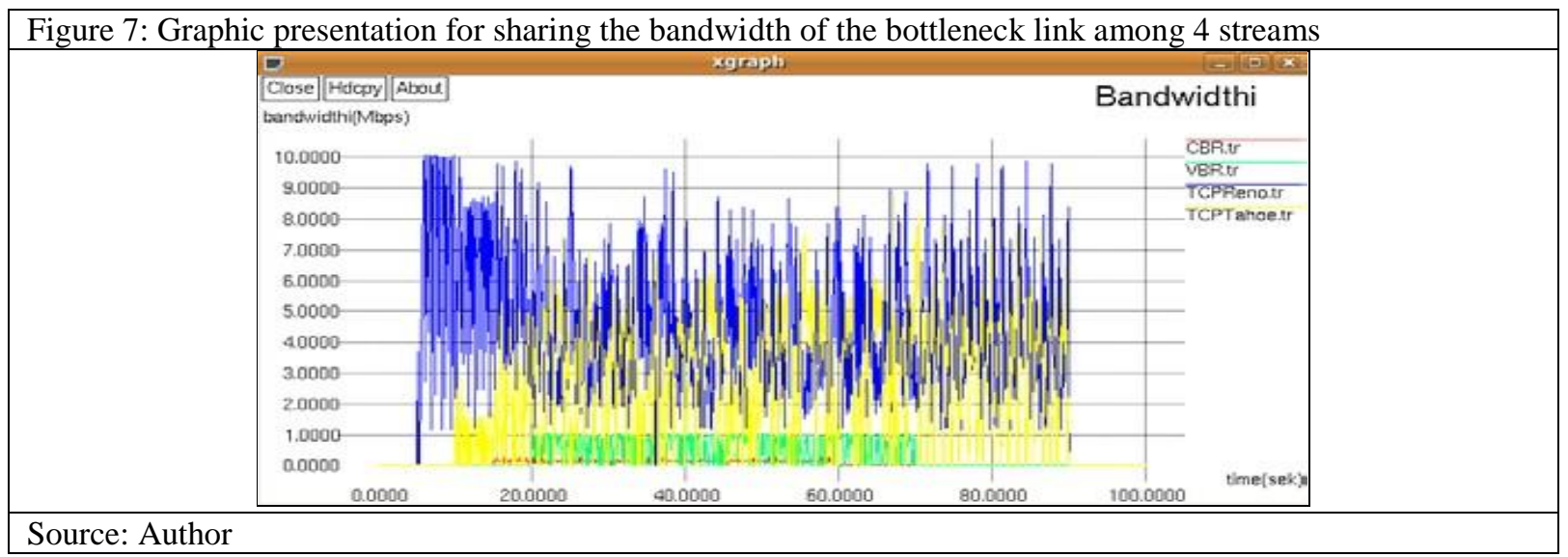


Figure 8: Graphic presentation for sharing the bandwidth of bottleneck link among 2 TCP streams

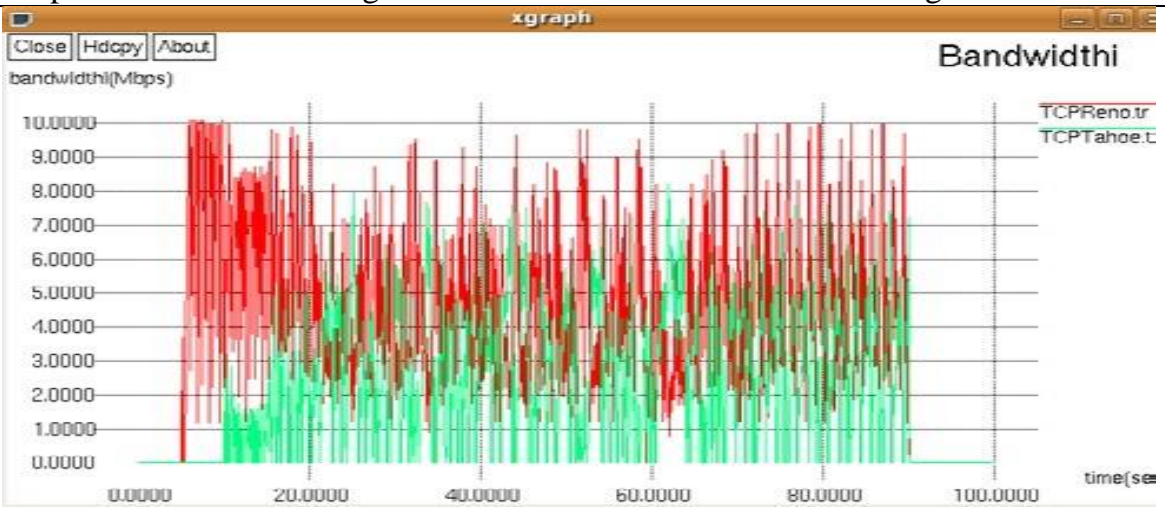

Source: Author

In the graphical representation of Figure 8 we note that in the presence of background traffic, the sharing of the bandwidth between streams starts to become fairer. This is due to the fact that with the increase of RTT, the delay of ranks also grows. With the increase of RTT, we see that there is also a decrease in the aggressiveness in getting the bandwidth for the TCP Reno stream, so TCP Tahoe has more opportunities to provide bandwidth even though the TCP Reno stream dominates on the network.

(Reminder: In telecommunications, the round-trip delay time (RTD) or round-trip time (RTT) is the length of time it takes for a signal to be sent plus the length of time it takes for an acknowledgment of that signal to be received. This time delay, therefore, consists of the propagation times between the two points of a signal).

\section{Calculation of the confidence interval}

The results of the simulation would not have any value if we don't have any idea about their accuracy, knowing that from every simulation a different result is received. To evaluate the data from every simulator, we must build the interval of confidence. To calculate the interval of confidence we proceed as shown below:

- We simulate and obtain an evaluation X1 for the measurement that we are interested

- We repeat the simulation M times and get X2...XM, all different from each other.

- We evaluate the average of $\mathrm{M}$ champions

$$
\bar{X}=\frac{\sum_{j=1}^{M} X_{j}}{M} \quad \sigma_{X}{ }^{2}=\frac{1}{M-1} \sum_{m=1}^{M}\left(X_{m}-\bar{X}\right)^{2}
$$

Knowing that we cannot find a right evaluation with only one value, we consider an interval [c1, c2]. Based on the theorem of certified limits, we assume that $95 \%$ of the values are in the same interval. For a Gaussian distribution, the interval of confidence evaluates with a derivation \pm 1.96 . The interval of confidence was $95 \%$

For $\mathrm{M}=10$ we get $\overline{\mathrm{X}}=2.17$ and $\sigma^{2} \mathrm{x} \quad$ accuracy is calculated with the following formula:

$$
\bar{X} \pm \frac{1.96 \sigma_{X}}{\sqrt{M}}
$$

Then we will calculate the interval of confidence for the bandwidth in the CBR stream.

For $\mathrm{M}=10$ we get $\overline{\mathrm{X}}=0.073$ and $\sigma 2 \mathrm{x}=0.020005$

The interval of confidence is $[0.069 ; 0.075]$. We calculate the interval of confidence for the bandwidth for the VBR stream:

For $\mathrm{M}=10$ we get $\mathrm{X}=0.29$ and $\sigma^{2} \mathrm{X}=0.0004$

The interval of confidence: [0.278; 0.302] We calculate the interval of confidence for the bandwidth 
in the TCP Reno stream: For $M=10$ we get $\overline{\mathrm{X}}=4.14$ and $\sigma \times 2=0.021$

The interval of confidence: $[2.081 ; 2.259]$

(as we stated above from the graphical appearances, the majority of the values are between 2-3 Mbps)

\section{Conclusion}

In this project, we experimented how TCP streams (TCP Reno and TCP Tahoe) compete with each other in the allocation of the bandwidth in a bottleneck link and also how they compete with background traffic (CBR and VBR). Traffic in the background ensures an approach that resembles more in a real world network, and it is noticed that with this presence on the network, TCP streams become more fair in relation to each other by reducing aggression. What was observed in the graphic presentations was that TCP Reno dominated in its transmission on the network compared to the standard TCP Tahoe. This refers to the fact that TCP Reno is an improvement of the standard TCP Tahoe because besides the algorithms that it uses - slow start, congestion avoidance, fast retransmit, it also uses the fast recovery algorithm. Fast Recovery acts in those cases when a certain number of duplicate ACKs are taken (threshold generally is set at 3). The sender retransmits the lost packets, but rather than the slow start algorithm, the congestion window is halved and then counts the duplicate ACKs for defining when to send the packages.

The interval of confidence: [4.053; 4.227] (as we stated above from the graphical appearance, the majority of the values are between 4-5 Mbps)

- We calculate the interval of confidence for the bandwidth for the TCP Tahoe stream:

- Starting from the graphical presentations, we affirm that TCP Reno uses on average $62 \%$ of the link bandwidth while TCP Tahoe $32 \%$. The rest of the link is shared between the CBR and VBR that use on average $1 \%$ and $5 \%$ of the available bandwidth of the bottleneck link.

TCP Reno performs better when the losses of packages are small. In cases where losses are great in a window, then Reno does not perform well, and its performance is almost the same as TCP Tahoe. Another problem is that if the window is very small when losses occur, then we will never get duplicate ACKs for fast retransmit and we must wait for timeouts. This method does not detect effectively the losses of packets.

\section{References}

Ha, S., Kim, Y., Le, L., Rhee, I. \&. Xu, L. (2011). A Step toward Realistic Performance Evaluation of High-Speed TCP Variants.

Cho, S. (2006). Congestion Control Schemes For Single And Parallel TCP Flows in High Bandwidth-Delay Product

Networks. Texas A\&M University.

Chohan, N. (2006). An Analysis of TCP through Simulation.

Issariyakul, T.A. (2009). Introduction to Network Simulator NS2.

Mo, J., La, J.R., Amatharam, V. \& Walrand, J. (1998). Analysis and Comparison of TCP Reno and TCP Vegas.

Yuvaraju, B.N \& Chiplunkar, N.N. (2010). Scenario Based Performance Analysis of Variants of TCP using NS2-Simulator. 